\title{
Quantum-Enhanced Advanced LIGO Detectors in the Era of Gravitational-Wave Astronomy
}

M. Tse $\odot,{ }^{1, *}$ Haocun Yu, ${ }^{1}$ N. Kijbunchoo, ${ }^{2}$ A. Fernandez-Galiana, ${ }^{1}$ P. Dupej, ${ }^{3}$ L. Barsotti®${ }^{1, \dagger}$ C. D. Blair, ${ }^{4}$ D. D. Brown, ${ }^{5}$ S. E. Dwyer, ${ }^{6}$ A. Effler, ${ }^{4}$ M. Evans, ${ }^{1}$ P. Fritschel, ${ }^{1}$ V. V. Frolov, ${ }^{4}$ A. C. Green, ${ }^{7}$ G. L. Mansell, ${ }^{1,6}$ F. Matichard ${ }^{8,1}$ N. Mavalvala, ${ }^{1}$ D. E. McClelland, ${ }^{2}$ L. McCuller, ${ }^{1}$ T. McRae, ${ }^{2}$ J. Miller, ${ }^{1}$ A. Mullavey, ${ }^{4}$ E. Oelker, ${ }^{1}$ I. Y. Phinney, ${ }^{1}$ D. Sigg, ${ }^{6}$ B. J. J. Slagmolen, ${ }^{2}$ T. Vo, ${ }^{9}$ R. L. Ward, ${ }^{2}$ C. Whittle, ${ }^{1}$ R. Abbott, ${ }^{8}$ C. Adams,${ }^{4}$ R. X. Adhikari, ${ }^{8}$ A. Ananyeva, ${ }^{8}$ S. Appert, ${ }^{8}$ K. Arai, ${ }^{8}$ J. S. Areeda, ${ }^{10}$ Y. Asali, ${ }^{11}$ S. M. Aston, ${ }^{4}$ C. Austin, ${ }^{12}$ A. M. Baer, ${ }^{13}$ M. Ball, ${ }^{14}$ S. W. Ballmer, ${ }^{9}$ S. Banagiri, ${ }^{15}$ D. Barker, ${ }^{6}$ J. Bartlett, ${ }^{6}$ B. K. Berger, ${ }^{16}$ J. Betzwieser, ${ }^{4}$ D. Bhattacharjee, ${ }^{17}$ G. Billingsley, ${ }^{8}$ S. Biscans, ${ }^{1,8}$ R. M. Blair, ${ }^{6}$ N. Bode,${ }^{18,19}$ P. Booker, ${ }^{18,19}$ R. Bork,${ }^{8}$ A. Bramley, ${ }^{4}$ A. F. Brooks, ${ }^{8}$ A. Buikema, ${ }^{1}$ C. Cahillane, ${ }^{8}$ K. C. Cannon, ${ }^{20}$ X. Chen, ${ }^{21}$ A. A. Ciobanu, ${ }^{5}$ F. Clara, ${ }^{6}$ S. J. Cooper,${ }^{22}$ K. R. Corley ${ }^{11}$ S. T. Countryman, ${ }^{11}$ P. B. Covas, ${ }^{23}$ D. C. Coyne, ${ }^{8}$ L. E. H. Datrier ${ }^{3}$ D. Davis, ${ }^{9}$ C. Di Fronzo, ${ }^{22}$ J. C. Driggers, ${ }^{6}$ T. Etzel,,${ }^{8}$ T. M. Evans, ${ }^{4}$ J. Feicht,${ }^{8}$ P. Fulda ${ }^{7}$ M. Fyffe, ${ }^{4}$ J. A. Giaime, ${ }^{12,4}$ K. D. Giardina, ${ }^{4}$ P. Godwin, ${ }^{24}$ E. Goetz, ${ }^{12,17}$ S. Gras, ${ }^{1}$ C. Gray,${ }^{6}$ R. Gray,${ }^{3}$ Anchal Gupta, ${ }^{8}$ E. K. Gustafson, ${ }^{8}$ R. Gustafson, ${ }^{25}$ J. Hanks, ${ }^{6}$ J. Hanson, ${ }^{4}$ T. Hardwick, ${ }^{12}$ R. K. Hasskew, ${ }^{4}$ M. C. Heintze, ${ }^{4}$ A. F. Helmling-Cornell, ${ }^{14}$ N. A. Holland, ${ }^{2}$ J. D. Jones, ${ }^{6}$ S. Kandhasamy, ${ }^{26}$ S. Karki, ${ }^{14}$ M. Kasprzack, ${ }^{8}$ K. Kawabe, ${ }^{6}$ P. J. King, ${ }^{6}$ J. S. Kissel, ${ }^{6}$ Rahul Kumar, ${ }^{6}$ M. Landry, ${ }^{6}$ B. B. Lane, ${ }^{1}$ B. Lantz, ${ }^{16}$ M. Laxen, ${ }^{4}$ Y. K. Lecoeuche, ${ }^{6}$ J. Leviton, ${ }^{25}$ J. Liu, ${ }^{18,19}$ M. Lormand, ${ }^{4}$ A. P. Lundgren, ${ }^{27}$ R. Macas, ${ }^{28}$ M. MacInnis, ${ }^{1}$ D. M. Macleod, ${ }^{28}$ S. Márka, ${ }^{11}$ Z. Márka, ${ }^{11}$ D. V. Martynov, ${ }^{22}$ K. Mason, ${ }^{1}$ T. J. Massinger, ${ }^{1}$ R. McCarthy, ${ }^{6}$ S. McCormick, ${ }^{4}$ J. McIver, ${ }^{8}$ G. Mendell, ${ }^{6}$ K. Merfeld, ${ }^{14}$ E. L. Merilh, ${ }^{6}$ F. Meylahn, ${ }^{18,19}$ T. Mistry, ${ }^{29}$ R. Mittleman, ${ }^{1}$ G. Moreno, ${ }^{6}$ C. M. Mow-Lowry, ${ }^{22}$ S. Mozzon, ${ }^{27}$ T. J. N. Nelson, ${ }^{4}$ P. Nguyen,,${ }^{14}$ L. K. Nuttall, ${ }^{28}$ J. Oberling, ${ }^{6}$ R. J. Oram, ${ }^{4}$ B. O'Reilly, ${ }^{4}$ C. Osthelder, ${ }^{8}$ D. J. Ottaway, ${ }^{5}$ H. Overmier, ${ }^{4}$ J. R. Palamos, ${ }^{14}$ W. Parker, ${ }^{4,30}$ E. Payne, ${ }^{31}$ A. Pele, ${ }^{4}$ C. J. Perez, ${ }^{6}$ M. Pirello, ${ }^{6}$ H. Radkins, ${ }^{6}$ K. E. Ramirez,${ }^{32}$ J. W. Richardson, ${ }^{8}$ K. Riles, ${ }^{25}$ N. A. Robertson, ${ }^{8,3}$ J. G. Rollins, ${ }^{8}$ C. L. Romel, ${ }^{6}$ J. H. Romie, ${ }^{4}$ M. P. Ross,${ }^{33}$ K. Ryan, ${ }^{6}$ T. Sadecki, ${ }^{6}$ E. J. Sanchez, ${ }^{8}$ L. E. Sanchez, ${ }^{8}$ T. R. Saravanan, ${ }^{26}$ R. L. Savage, ${ }^{6}$ D. Schaetzl, ${ }^{8}$ R. Schnabel, ${ }^{34}$ R. M. S. Schofield, ${ }^{14}$ E. Schwartz, ${ }^{4}$ D. Sellers, ${ }^{4}$ T. J. Shaffer, ${ }^{6}$ J. R. Smith, ${ }^{10}$ S. Soni,${ }^{12}$ B. Sorazu, ${ }^{3}$ A. P. Spencer, ${ }^{3}$ K. A. Strain, ${ }^{3}$ L. Sun, ${ }^{8}$ M. J. Szczepańczyk, ${ }^{7}$ M. Thomas, ${ }^{4}$ P. Thomas, ${ }^{6}$ K. A. Thorne, ${ }^{4}$ K. Toland, ${ }^{3}$ C. I. Torrie, ${ }^{8}$ G. Traylor, ${ }^{4}$ A. L. Urban, ${ }^{12}$ G. Vajente, ${ }^{8}$ G. Valdes,${ }^{12}$ D. C. Vander-Hyde, ${ }^{9}$ P. J. Veitch, ${ }^{5}$ K. Venkateswara, ${ }^{33}$ G. Venugopalan, ${ }^{8}$ A. D. Viets, ${ }^{35}$ C. Vorvick, ${ }^{6}$ M. Wade ${ }^{36}$ J. Warner, ${ }^{6}$ B. Weaver, ${ }^{6}$ R. Weiss, ${ }^{1}$ B. Willke, ${ }^{18,19}$ C. C. Wipf,${ }^{8}$ L. Xiao, ${ }^{8}$ H. Yamamoto, ${ }^{8}$ M. J. Yap, ${ }^{2}$ Hang Yu, ${ }^{1}$ L. Zhang, ${ }^{8}$ M. E. Zucker, ${ }^{1,8}$ and J. Zweizig ${ }^{8}$

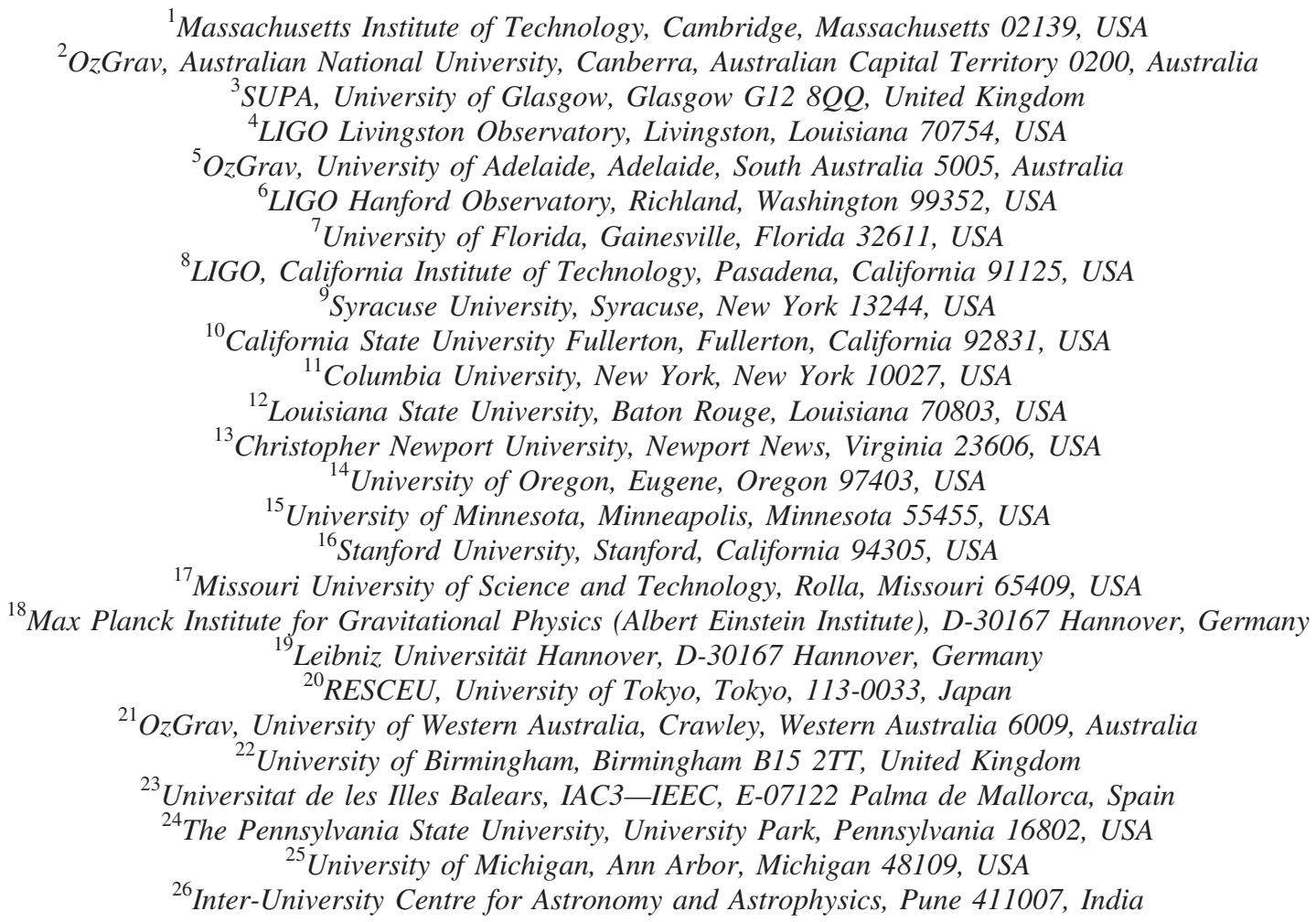




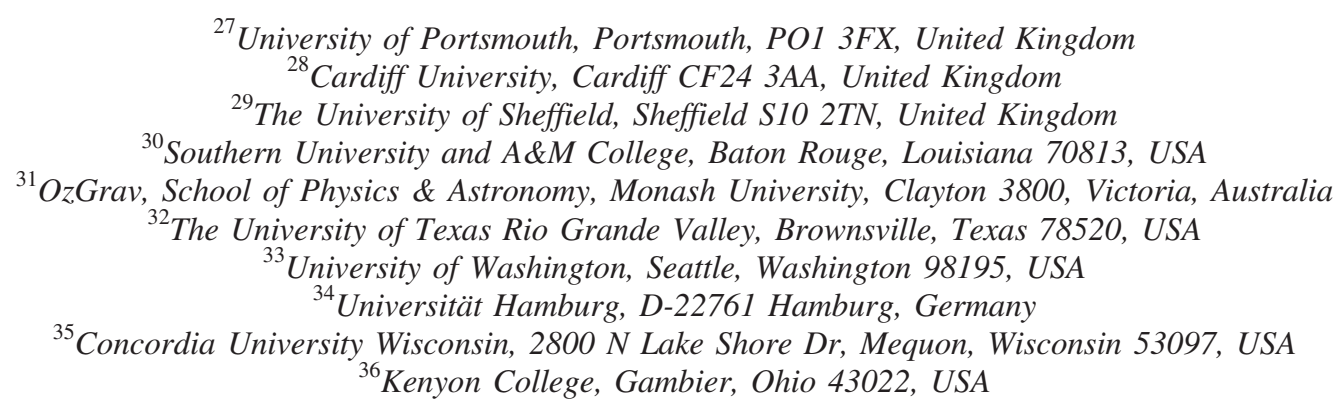

(Received 4 October 2019; published 5 December 2019)

\begin{abstract}
The Laser Interferometer Gravitational Wave Observatory (LIGO) has been directly detecting gravitational waves from compact binary mergers since 2015. We report on the first use of squeezed vacuum states in the direct measurement of gravitational waves with the Advanced LIGO H1 and L1 detectors. This achievement is the culmination of decades of research to implement squeezed states in gravitational-wave detectors. During the ongoing O3 observation run, squeezed states are improving the sensitivity of the LIGO interferometers to signals above $50 \mathrm{~Hz}$ by up to $3 \mathrm{~dB}$, thereby increasing the expected detection rate by $40 \%$ (H1) and $50 \%$ (L1).
\end{abstract}

DOI: 10.1103/PhysRevLett.123.231107

\section{INTRODUCTION}

The Advanced LIGO detectors opened the era of gravitational-wave astronomy in 2015 , with the first detection of gravitational waves from a binary black hole system [1]. By the end of the second observing run $(\mathrm{O} 2)$ in August 2017, a total of 11 confirmed gravitational-wave signals have been reported by the LIGO and Virgo Collaborations [2], including one from a binary neutron star merger [3]. A third observing run (O3) started on April 1, 2019, with improved LIGO and Virgo detectors [4,5].

The Advanced LIGO instruments (the L1 detector in Livingston, Louisiana and the $\mathrm{H} 1$ detector in Hanford, Washington) are dual-recycled Michelson interferometers with Fabry-Perot cavities in the arms [6,7]. They measure gravitational-wave strain as arm-length modulations of the orthogonal 4-km long arms.

Quantum noise is one of the fundamental limitations to the sensitivity of gravitational wave detectors and it manifests in two ways: quantum shot noise, caused by statistical fluctuations in the arrival time of photons at the interferometer output; and quantum radiation pressure noise, due to quantum fluctuations in the photon flux impinging on the interferometer mirrors [8].

Decades of world-wide research have targeted improvements to the quantum-limited sensitivity of gravitationalwave detectors [9]. Injecting squeezed vacuum states has

Published by the American Physical Society under the terms of the Creative Commons Attribution 4.0 International license. Further distribution of this work must maintain attribution to the author(s) and the published article's title, journal citation, and DOI. emerged as a parallel technique to increasing laser power [10], with routine use in the GEO interferometer [11,12] and a proof-of-principle experiment in the initial LIGO H1 detector [13].

In this Letter we present the first implementation of squeezed vacuum states in the Advanced LIGO detectors. This result is remarkable not only because squeezing significantly improves the sensitivity of Advanced LIGO and is now routinely used for detecting gravitational waves $[14,15]$; it is also the first time that squeezing is used in a dual-recycled Michelson interferometer with Fabry-Perot cavities in the arms [16], the baseline optical configuration of all of the next generation instruments currently under consideration $[17,18]$.

We also acknowledge a historical milestone. The concept of squeezed vacuum states for improving the sensitivity of gravitational-wave detectors was invented nearly four decades ago [19]. This work marks the first direct observation of gravitational waves with quantum-enhanced interferometers. Here we describe the unique features of the Advanced LIGO squeezed vacuum source, as well as the characterization of its performance and prospects for further improvement.

\section{RESULTS}

Both the L1 and H1 Advanced LIGO interferometers operate with squeezed vacuum states during $\mathrm{O} 3$.

Figure 1 shows the L1 strain noise amplitude spectral density with and without squeezing (in green and black, respectively). The improvement is $35 \%$ at $1.1-1.4 \mathrm{kHz}$ (a frequency band with minimal impact from nonquantum noises), with squeezing enhancement visible down to 


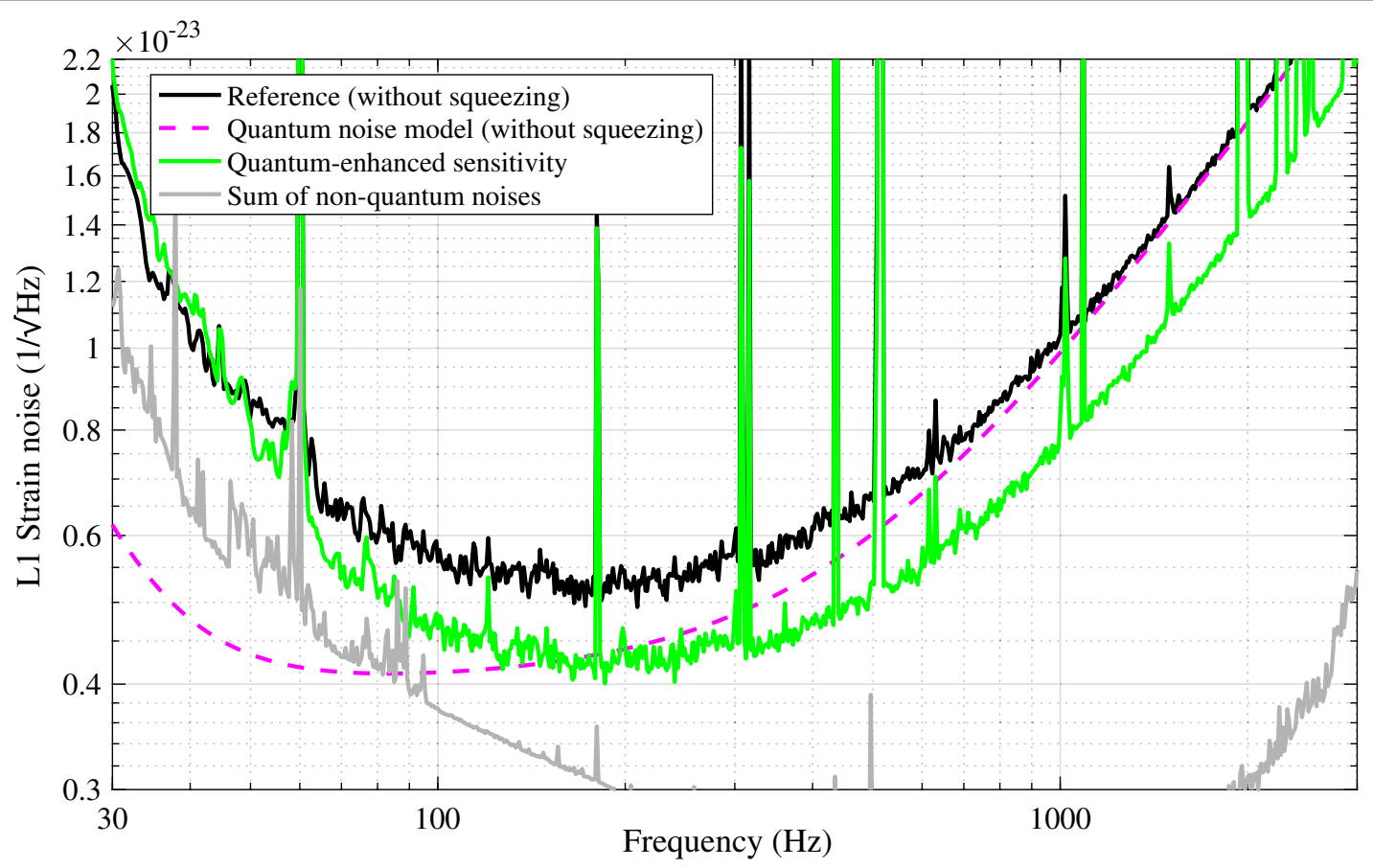

FIG. 1. The green trace shows the typical L1 strain amplitude spectral density taken in April 2019 during O3, and the black trace gives a reference showing the spectrum without squeezing. The squeezing spectrum is taken with the $\mathrm{O} 3$ observing configuration, which includes a reduced nonlinear gain and an optimized squeezed quadrature angle, in order to maximize binary neutron star range. The purple trace shows a model of the quantum noise for the reference configuration, while the sum of all of the remaining known (nonquantum) noises is shown in gray (the methods used to estimate known noise sources are detailed in Ref. [20], with O3 measurements detailed in Ref. [4]). After subtracting nonquantum noises, analysis of these spectra show $2.7 \pm 0.1 \mathrm{~dB}$ of shot-noise squeezing at $1.1-1.4 \mathrm{kHz}$ in typical operation, whereas the maximum achieved shot-noise squeezing level in the interferometer is $3.2 \pm 0.1 \mathrm{~dB}$.

$50 \mathrm{~Hz}$. The H1 spectra look qualitatively similar, although the noise reduction is lower, at $25 \%$.

We note that to achieve the same reduction in shot noise would require $85 \%$ (L1) and 65\% (H1) more laser power, which is beyond the capability of the current laser system. Moreover, increasing the laser power complicates the control of the interferometer due to thermal effects [21], angular instabilities caused by photon radiation-pressure induced torques [22], and parametric instabilities [23].

A primary figure of merit of a gravitational-wave detector is its binary-neutron-star (BNS) range [24], a distance representing the detector sensitivity [25]. To optimize this range, the squeezing parameters are tuned to balance the increase of quantum radiation pressure noise with the benefit of reduced quantum shot noise [26]. Although up to $3.2 \mathrm{~dB}$ of squeezing could be observed in the $\mathrm{L} 1$ detector, $2.7 \mathrm{~dB}$ are used in normal operations, thereby improving the BNS range by $14 \%$. A squeezing level of $2.0 \mathrm{~dB}$ in $\mathrm{H} 1$ improves its BNS range by $12 \%$. The expected detection rate is increased by $50 \%$ in $\mathrm{L} 1$ and $40 \%$ in $\mathrm{H} 1$, as the rate scales with the volume of the observed Universe.

Squeezing has been used throughout the first six months of O3. In principle, a movable diverter can isolate the interferometer from the squeezed vacuum source, thus allowing observation at nonenhanced sensitivity without degrading the overall detector duty cycle. This option is rarely needed however, as the squeezing system has been robust, enhancing sensitivity during $98 \%$ of the detector observation time in L1 and $100 \%$ in $\mathrm{H} 1$. Dozens of interesting gravitational-wave candidates have been reported in this period [14], and, in particular, a BNS event (GW190425 [15]) was detected by the squeezingenhanced L1 detector.

\section{INSTRUMENTATION FOR THE ADVANCED LIGO SQUEEZING SYSTEM}

The sensitivity of the Advanced LIGO detector places strict requirements on the performance of the squeezed vacuum source [10]. A process known as backscatterwhere stray light from the main interferometer field scatters towards moving surfaces, acquires phase fluctuations, and scatters back toward the interferometer readout-can degrade the interferometer sensitivity [27]. To prevent this effect, the Advanced LIGO squeezer has a unique design that places core components on a seismically isolated platform housed inside the main LIGO vacuum envelope $[28,29]$. Combined with a bowtie geometry for the optical parametric oscillator (OPO) $[30,31]$ that generates the squeezing, and the use of an additional Faraday isolator on the squeezed vacuum injection path, the system provides a backscatter isolation ratio measured to be $130 \mathrm{~dB}$, which 


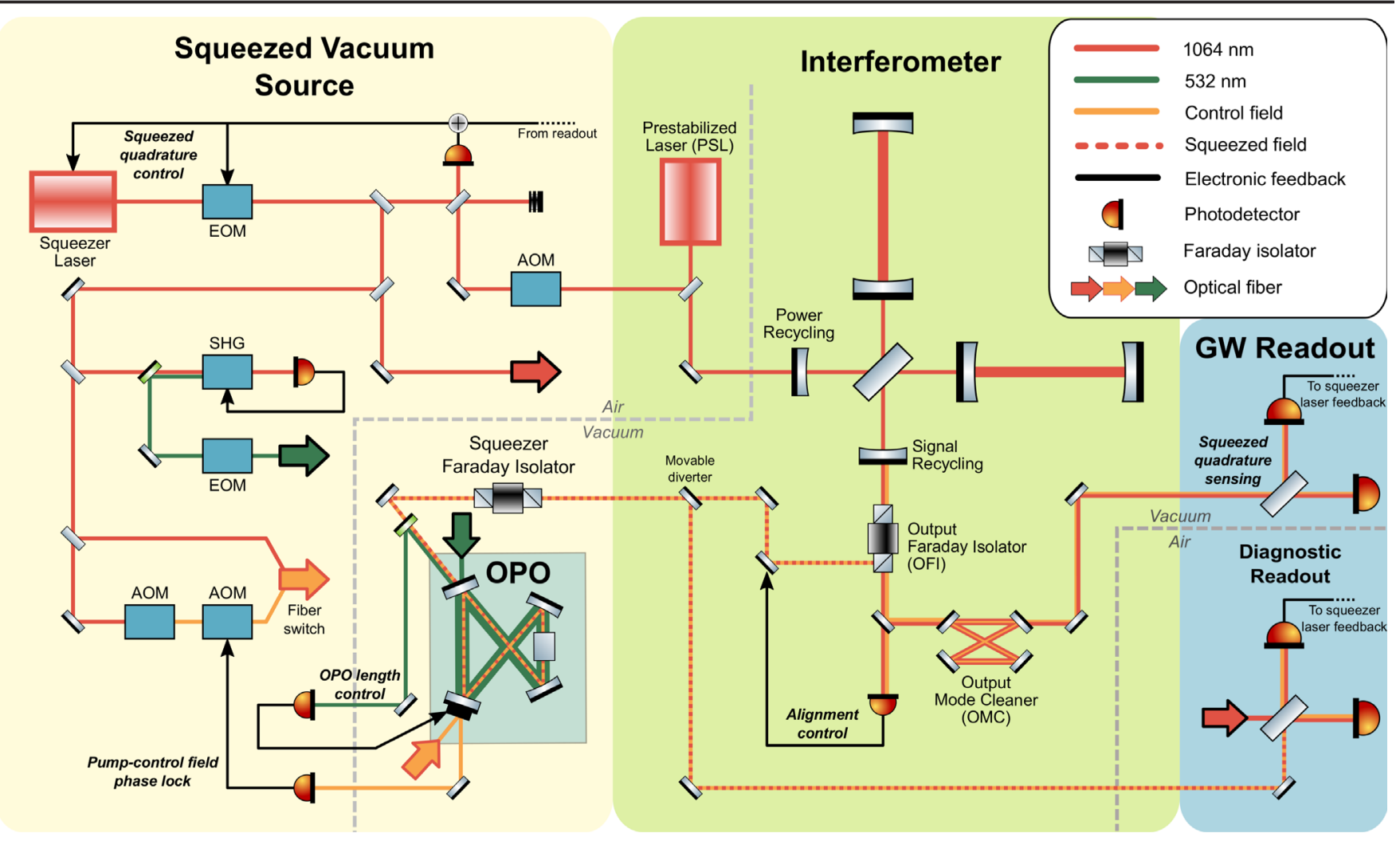

FIG. 2. Conceptual layout of the squeezed vacuum subsystem for Advanced LIGO. A continuous 1W Nd:YAG laser at 1064 nm, phase locked to the prestabilized laser (PSL), is used to derive three fields that are launched into fibers: (i) a pump field (green arrow) produced by a second harmonic generator (SHG) and imprinted with $80 \mathrm{MHz}$ sidebands, by an electro-optic modulator (EOM), for feedback control of the optical parametric oscillator (OPO) length via Pound-Drever-Hall sensing; (ii) a control field (orange arrow), produced by two acousto-optical modulators (AOMs), used for sensing the phase of the squeezed vacuum field (when needed, the fiber switch here is toggled to launch a $1064 \mathrm{~nm}$ diagnostic field in place of the control field, to measure nonlinear gain and losses); and (iii) a local oscillator (red arrow) used for diagnostic homodyne measurements. In typical operation, the squeezed field propagates to the gravitational-wave $(\mathrm{GW})$ readout. A remotely controlled movable mirror provides the option of diverting the squeezed field out of the vacuum chamber, enabling measurements of nonsqueezed reference spectra in the interferometer as shown in Fig. 1, as well as diagnostic measurements using the homodyne detector [33].

is consistent with the design requirement [29]. The fields used to operate this in-vacuum OPO are generated in air and delivered to the OPO via optical fibers [32]. The output ends of these fibers are fastened to the suspended platform of the OPO, providing the benefit of long-term alignment stability.

Figure 2 shows the optical and electronic setup for generating and controlling squeezed light. The central component is a subthreshold OPO, a traveling-wave cavity built around a periodically poled potassium titanyl phosphate (PPKTP) crystal. A second-order nonlinearity in PPKTP allows interaction between a $532 \mathrm{~nm}$ pump field and the vacuum fluctuations of the $1064 \mathrm{~nm}$ field. The resulting squeezed vacuum state is steered via two suspended mirrors [34] to the output Faraday isolator (OFI), which circulates the beam back towards the antisymmetric port of the interferometer.

To control the phase of the squeezed vacuum field, a control field consisting of a single rf sideband at $+3.1 \mathrm{MHz}$ relative to the main laser frequency is injected into the OPO $[35,36]$. This sideband is generated by two AOMs in series, which sequentially shift the frequency of the input $1064 \mathrm{~nm}$ field down by $200 \mathrm{MHz}$ and then up by $203.1 \mathrm{MHz}$ to create $\mathrm{a}+3.1 \mathrm{MHz}$ sideband. This technique obviates the need for a second laser and phase lock $[32,37]$. Through the parametric interaction between the $+3.1 \mathrm{MHz}$ sideband and the $532 \mathrm{~nm}$ pump field, the OPO generates a $-3.1 \mathrm{MHz}$ sideband, and the $6.2 \mathrm{MHz}$ beat note between the injected upper and generated lower sidebands is fed back to one of the AOMs to lock the relative phase of the pump and control fields. The squeezed vacuum state generated in the OPO is in phase with the pump field, so the control field, which lies well within the linewidth of the OPO $(24.5 \mathrm{MHz})$, can be used as a proxy for sensing the phase of the squeezed vacuum field.

In full operation, the control field copropagates with the squeezed field, and its beat note with the main interferometer field is sensed at the main readout photodetector in 
TABLE I. Predicted squeezing and antisqueezing levels for the L1 interferometer, with nonlinear gain $g=2.9 \pm 0.1$ : (a) generated levels at the OPO, (b) levels considering only losses, (c) levels considering losses and phase noise. *These are estimates informed by indirect measurements, as direct measurements of these factors are not available.

\begin{tabular}{llc}
\hline \hline (a) Generated squeezing & \multicolumn{1}{c}{ OPO parameter } & Value \\
\hline$\left(\eta=0.98, \theta_{\text {rms }}=0\right.$ mrad $)$ & Measured nonlinear gain $g$ & $2.9 \pm 0.1$ \\
$7.2 \pm 0.3 \mathrm{~dB}$ squeezing & OPO escape efficiency & $0.98 \pm 0.01$ \\
$7.6 \pm 0.2 \mathrm{~dB}$ antisqueezing &
\end{tabular}

\begin{tabular}{llr}
\hline \hline$(\mathrm{b})+$ Losses & \multicolumn{1}{c}{ Loss source } & Efficiency \\
\hline$\left(\eta=0.75, \theta_{\text {rms }}=0\right.$ mrad $)$ & Optics OPO to OFI & $0.95 \pm 0.01$ \\
$4.1 \pm 0.6 \mathrm{~dB}$ squeezing & OFI double-pass & $0.94 \pm 0.01$ \\
$6.5 \pm 0.4 \mathrm{~dB}$ antisqueezing & OMC transmission & $0.95 \pm 0.01$ \\
& Photodiode efficiency & $0.98 \pm 0.01$ \\
& Alignment and mode matching* & $0.90 \pm 0.05$ \\
& Total efficiency $\eta$ & $0.75 \pm 0.06$ \\
\hline \hline
\end{tabular}

\begin{tabular}{llc}
\hline \hline (c) + Losses + phase noise & Phase noise source & Value $(\mathrm{mrad})$ \\
\hline$\left(\eta=0.75, \theta_{\text {rms }}=\sim 15 \mathrm{mrad}\right)$ & Phase lock residual & 10.6 \\
$4.0 \pm 0.6 \mathrm{~dB}$ squeezing & rf sidebands* & 10 \\
$6.5 \pm 0.4 \mathrm{~dB}$ antisqueezing & Total phase noise $\theta_{\text {rms }}$ & $\sim 15$ \\
\hline \hline
\end{tabular}

transmission of the output mode cleaner (OMC) [12]. This signal is then fed back to the main squeezer laser to lock the squeezed quadrature angle for injecting high-frequency squeezing $\left(\phi=\phi_{0}\right)$, antisqueezing $\left(\phi=\phi_{0}+\pi / 2\right)$, or any mixture of the two. The alignment control signals are generated at a pickoff before the OMC [38] from the beat note between the control field and the $45 \mathrm{MHz}$ sideband of the main interferometer field.

\section{CHARACTERIZATION}

The squeezing level measured in the interferometer is determined by the amount of generated squeezing (as quantified by the OPO nonlinear gain), and by the optical losses and phase noise in the system. Nonlinear gain, losses, and phase noise can be measured or budgeted independently to predict an expected squeezing level, as shown in Table I. In addition, they can be inferred by measuring the squeezed and antisqueezed quadratures, since losses and phase noise affect the magnitude of observed squeezing and antisqueezing in different ways [see Eqs. (1),(2) in Ref. [32] ]. Here we characterize the L1 squeezed vacuum source by measuring squeezing and antisqueezing while operating the $\mathrm{OPO}$ at a nonlinear gain of $g=2.9 \pm 0.1$, and compare the inferred losses and phase noise to the budgeted levels.

\section{A. Nonlinear gain}

The nonlinear gain $g$ represents the strength of interaction in the OPO between the $532 \mathrm{~nm}$ pump field and the
$1064 \mathrm{~nm}$ vacuum fluctuations. It is a function of the pump power and OPO cavity parameters, and determines the amount of squeezing generated by the OPO. It can be directly measured by injecting a $1064 \mathrm{~nm}$ diagnostic field through the OPO in place of the control field, via a fiber switch (see Fig. 2). Then $g$ is the ratio of transmitted power though the OPO with and without nonlinear gain (the OPO can be detuned in temperature and pump power so that $g=1$ ).

\section{B. Losses}

Optical loss can usually be measured by observing the attenuation of a $1064 \mathrm{~nm}$ diagnostic field along the path of squeezing injection. However, loss sources such as misalignment and mode-mismatch change depending on the thermal state of the interferometer. For these sources, an estimate is provided in Table I. Accounting for all known optical losses, we estimate the total efficiency of the system to be 0.75 .

\section{Phase noise}

While the relative phase between the squeezed field and the main interferometer field is controlled through a series of feedback loops, residual fluctuations known as phase noise affect squeezing measurements [39]. Fluctuations at frequencies higher than the measurement frequency will mix antisqueezing in to a squeezing measurement and vice versa, effectively degrading the observable level of squeezing. The dominant source of phase noise is expected to be the vibration of fibers and optical components causing 
fluctuations in optical path length, and is mitigated through the use of a rigid, nearly monolithic OPO and the careful acoustic isolation of fibers and optics [32]. We independently estimate the level of phase noise by summing the residual fluctuations seen by the phase-lock loop between the control field and the $532 \mathrm{~nm}$ pump field, and the phase-lock loop between the control field and the main interferometer field. The rf sidebands of the main interferometer also add phase noise that is estimated separately [39]. Summing the above contributions in quadrature, we estimate the total rms phase noise in the system to be $15 \mathrm{mrad}$.

\section{Squeezing}

Combining the direct measurements of nonlinear gain, losses, and phase noise, we arrive at a predicted squeezing level of $4.0 \pm 0.6 \mathrm{~dB}$ with $6.5 \pm 0.4 \mathrm{~dB}$ antisqueezing. The squeezing level we observe at $1.1-1.4 \mathrm{kHz}$ is $3.2 \pm 0.1 \mathrm{~dB}$, and the antisqueezing level, as measured in the same frequency band by rotating the squeezed quadrature angle by $90^{\circ}$, is $6.1 \pm 0.1 \mathrm{~dB}$. These results indicate the efficiency of the system to be $0.66 \pm 0.02$. This differs from the budgeted loss by about $10 \%$, with the discrepancy most likely due to mode matching and misalignment losses being higher than estimated. We note that at $100-200 \mathrm{~Hz}$, the observed total loss is consistent with the predicted loss. Although nonquantum noises are at a comparable level to quantum noise in this frequency region, this observation still suggests the presence of a frequency-dependent loss mechanism $[40,41]$.

The measured squeezing and antisqueezing levels are consistent with the budgeted phase noise, but the observed phase noise is weakly constrained at this nonlinear gain, to below $80 \mathrm{mrad}$. Future tests will prioritize increasing the nonlinear gain further in order to better constrain the phase noise.

While further characterization and optimization for the H1 system are still ongoing, the goals of increasing nonlinear gain, reducing losses, and better characterizing phase noise are common between the two sites.

\section{OPTIMIZATION}

Two main aspects make squeezing injection in the Advanced LIGO interferometers unique with respect to previous applications: the use of a signal-recycling cavity (SRC) introduces frequency-dependent effects on the measured squeezing level and the high circulating power in the Fabry-Perot arms renders quantum radiation pressure noise non-negligible.

\section{A. SRC length tuning}

The signal recycling cavity provides the option of tuning the frequency response of the interferometer [6]. Although typical operation in $\mathrm{O} 3$ calls for a zero detuning of the signal recycling cavity length to achieve broadband interferometer response to gravitational waves, any unintentional offset adds a frequency-dependence to the squeezed vacuum sidebands. To set up the interferometer for observation, we tune the signal-recycling cavity length while injecting squeezing to achieve the same level of squeezing across the entire shot-noise limited frequency band.

\section{B. Quantum radiation pressure noise}

The L1 interferometer operates with approximately $200 \mathrm{~kW}$ of circulating power in the arms [4]. Although quantum radiation pressure noise is not a dominant noise source, the increase of this noise due to antisqueezed vacuum is still relevant [26]. In small-scale systems, this noise has been observed [42] and reduced via squeezing [43]. Here, we lower the nonlinear gain to $g=2.7 \pm 0.1$ to reduce quantum radiation pressure noise for the $\mathrm{O} 3$ observing configuration. Even with a reduced nonlinear gain, the squeezed quadrature angle that optimizes BNS detection range, $\phi_{\text {optimum }}$, is not necessarily the angle that maximizes shot noise squeezing, $\phi_{0}$. We find that the difference is $\Delta \phi=7^{\circ}$, so the squeezer is operated at this squeeze angle during $\mathrm{O} 3$. This represents the injection of a carefully calibrated mixture of squeezing and antisqueezing to balance high-frequency performance against low-frequency quantum radiation pressure noise.

Figure 1 shows the L1 strain noise in this optimized configuration (a nonlinear gain of $g=2.7 \pm 0.1$, a squeezed quadrature angle offset of $\Delta \phi=7^{\circ}$, and a tuned signal recycling cavity length), compared to a reference measured without squeezing. This configuration gives us $2.7 \pm 0.1 \mathrm{~dB}$ of squeezing at $1.1-1.4 \mathrm{kHz}$. A model of the quantum noise for the reference configuration is also shown, as well as the sum of all of the known nonquantum noises present in the system (as described in Ref. [4]). For technical reasons, the $\mathrm{H} 1$ interferometer has operated with a lower nonlinear gain of $g=2.3 \pm 0.1$ (and thus without squeezed quadrature angle offset), with $2.0 \pm 0.1 \mathrm{~dB}$ of squeezing measured.

\section{CONCLUSIONS AND OUTLOOK}

Nearly 40 years after squeezing was first conceived as a way of improving the sensitivity of gravitational wave detectors, both the Livingston and Hanford interferometers operate with squeezed vacuum states during the $\mathrm{O} 3$ observing run. Squeezing enhances the sensitivity of the L1 Advanced LIGO detector by $2.7 \mathrm{~dB}$ in its shot-noise limited frequency region, above $50 \mathrm{~Hz}$, leading to a $14 \%$ increase in BNS range and 50\% increase in expected detection rate. The squeezing-enhanced L1 detector has achieved the highest BNS range ever reported, up to $140 \mathrm{Mpc}$. For the Hanford detector, the improvement of $2.0 \pm 0.1 \mathrm{~dB}$ gives a $12 \%$ increase in BNS range, up to $120 \mathrm{Mpc}$, with optimization of the system still ongoing. 
The path forward for further squeezing enhancement is twofold: (i) higher levels of squeezing are within reach by further reducing the optical loss in the system, and (ii) frequency-dependent squeezing will be crucial for a broadband reduction of quantum noise [44]. The upgrade of the Advanced LIGO detectors known as "A+" targets both of these improvements by coupling the squeezed source described in this Letter with a narrow linewidth filter cavity to achieve frequency-dependent squeezing [45-47]. Moreover, lower loss Faraday isolators [48] and active mode matching elements will be adopted to optimize the mode matching between the squeezed beam and the interferometer beam. With these improvements, A+ aims for a $6 \mathrm{~dB}$ broadband reduction in quantum noise [49].

In addition to the direct impact on the ability to detect astrophysical sources, reduced quantum noise enhances the science that can be done with the sources that are detected. In particular, our ability to localize sources on the sky depends on the instrument's high frequency sensitivity, where squeezing has a large impact [50]. Also, information about the tidal deformability and interior structure of neutron stars is contained in the high frequency pre- and postmerger gravitational-wave signals. It has been shown that for a system similar to the binary-neutron star GW170817 [51], the post-merger signal could be extracted with the squeezed-enhanced $\mathrm{A}+$ detectors.

\section{ACKNOWLEDGMENTS}

LIGO was constructed by the California Institute of Technology and Massachusetts Institute of Technology with funding from the National Science Foundation, and operates under Cooperative Agreement No. PHY-0757058. Advanced LIGO was built under Grant No. PHY-0823459. The authors also gratefully acknowledge the support of the Australian Research Council under the ARC Centre of Excellence for Gravitational Wave Discovery, Grant No. CE170100004 and Linkage Infrastructure, Equipment and Facilities Grant No. LE170100217; the National Science Foundation Graduate Research Fellowship under Grant No. 1122374; the Science and Technology Facilities Council of the United Kingdom, and the LIGO Scientific Collaboration Fellows program.

*maggie.tse@ligo.org

${ }^{\dagger}$ lisa.barsotti@ligo.org

[1] B. P. Abbott et al. (LIGO Scientific and Virgo Collaborations), Phys. Rev. Lett. 116, 061102 (2016).

[2] B. P. Abbott et al. (LIGO Scientific and Virgo Collaborations), Phys. Rev. X 9, 031040 (2019).

[3] B. P. Abbott et al. (LIGO Scientific and Virgo Collaborations), Phys. Rev. Lett. 119, 161101 (2017).

[4] A. Buikema et al. (to be published).

[5] F. Acernese et al. (Virgo Collaboration) and H. Vahlbruch et al., following Letter, Phys. Rev. Lett. 123, 231108 (2019).
[6] J. Aasi et al. (LIGO Scientific Collaboration), Classical Quantum Gravity 32, 074001 (2015).

[7] B. P. Abbott et al. (LIGO Scientific and Virgo Collaborations), Phys. Rev. Lett. 116, 131103 (2016).

[8] C. M. Caves, Phys. Rev. Lett. 45, 75 (1980).

[9] D. E. McClelland, N. Mavalvala, Y. Chen, and R. Schnabel, Laser Photonics Rev. 5, 677 (2011).

[10] L. Barsotti, J. Harms, and R. Schnabel, Rep. Prog. Phys. 82, 016905 (2018).

[11] LIGO Scientific Collaboration, Nat. Phys. 7, 962 (2011).

[12] H. Grote, K. Danzmann, K. L. Dooley, R. Schnabel, J. Slutsky, and H. Vahlbruch, Phys. Rev. Lett. 110, 181101 (2013).

[13] J. Aasi et al., Nat. Photonics 7, 613 (2013).

[14] LIGO and Virgo Collaborations, https://gracedb.ligo.org/ latest/.

[15] LIGO and Virgo Collaborations, https://gen.gsfc.nasa.gov/ gen3/24168.gcn3.

[16] A. Buonanno and Y. Chen, Phys. Rev. D 64, 042006 (2001).

[17] M. Punturo et al., Classical Quantum Gravity 27, 194002 (2010).

[18] B. P. Abbott et al. (LIGO Scientific Collaboration), Classical Quantum Gravity 34, 044001 (2017).

[19] C. Caves, Phys. Rev. D 23, 1693 (1981).

[20] D. V. Martynov et al., Phys. Rev. D 93, 112004 (2016).

[21] A. F. Brooks, D. Hosken, J. Munch, P. J. Veitch, Z. Yan, C. Zhao, Y. Fan, L. Ju, D. Blair, P. Willems, B. Slagmolen, and J. Degallaix, Appl. Opt. 48, 355 (2009).

[22] K. L. Dooley, L. Barsotti, R. X. Adhikari, M. Evans, T. T. Fricke, P. Fritschel, V. Frolov, K. Kawabe, and N. SmithLefebvre, J. Opt. Soc. Am. A 30, 2618 (2013).

[23] M. Evans, S. Gras, P. Fritschel, J. Miller, L. Barsotti et al., Phys. Rev. Lett. 114, 161102 (2015).

[24] H. Y. Chen, D. E. Holz, J. Miller, M. Evans, S. Vitale, and J. Creighton, arXiv:1709.08079.

[25] The BNS range corresponds to the radius of the sensitive volume of the Universe for 1.4-1.4 $M_{\odot}$ neutron star binary systems (assuming a detection threshold with matched-filter signal-to-noise ratio of 8 in a single detector), integrated over the interferometer antenna pattern, and averaged over all binary inclinations and orientations. Although BNS signals end above a $\mathrm{kHz}$ and typical $\mathrm{BBH}$ signals end around $500 \mathrm{~Hz}$, both types of signals accumulate most of their SNR in the $30-300 \mathrm{~Hz}$ band. Thus the impact of squeezing on detector range is similar for both $\mathrm{BNS}$ and $\mathrm{BBH}$.

[26] H. Yu et al. (to be published).

[27] S. S. Y. Chua et al., Classical Quantum Gravity 31, 035017 (2014).

[28] A. Fernandez-Galiana et al., arXiv:1901.09666.

[29] E. Oelker, L. Barsotti, S. Dwyer, D. Sigg, and N. Mavalvala, Opt. Express 22, 21106 (2014).

[30] A. Wade, G. L. Mansell, S. S. Y. Chua, Robert L. Ward, Bram J. J. Slagmolen, Daniel A. Shaddock, and David E. McClellandet al., Sci. Rep. 5, 18052 (2015).

[31] S. S. Y. Chua, M. S. Stefszky, C. M. Mow-Lowry, B. C. Buchler, S. Dwyer, D. A. Shaddock, P. K. Lam, and D. E. McClelland, Opt. Lett. 36, 4680 (2011).

[32] E. Oelker, G. Mansell, M. Tse, J. Miller, F. Matichard, L. Barsotti, P. Fritschel, D. E. McClelland, M. Evans, and N. Mavalvala, Optica 3, 682 (2016). 
[33] M. S. Stefszky, C. M. Mow-Lowry, S. S. Y. Chua, D. A. Shaddock, B. C. Buchler, H. Vahlbruch, A. Khalaidovski, R. Schnabel, P. K. Lam, and D. E. McClelland, Classical Quantum Gravity 29, 145015 (2012).

[34] B. J. J. Slagmolen, A. J. Mullavey, J. Miller, D. E. McClelland, and P. Fritschel, Rev. Sci. Instrum. 82, 125108 (2011).

[35] H. Vahlbruch, S. Chelkowski, B. Hage, A. Franzen, K. Danzmann, and R. Schnabel, Phys. Rev. Lett. 97, 011101 (2006).

[36] S. Chelkowski, H. Vahlbruch, K. Danzmann, and R. Schnabel, Phys. Rev. A 75, 043814 (2007).

[37] M. Tse et al. (to be published).

[38] E. Schreiber, K. L. Dooley, H. Vahlbruch, C. Affeldt, A. Bisht, J. R. Leong, J. Lough, M. Prijatelj, J. Slutsky, M. Was, H. Wittel, K. Danzmann, and H. Grote, Opt. Express 24, 146 (2016).

[39] S. Dwyer et al., Opt. Express 21, 19047 (2013).

[40] P. Kwee, J. Miller, T. Isogai, L. Barsotti, and M. Evans, Phys. Rev. D 90, 062006 (2014).

[41] L. McCuller et al. (to be published).
[42] J. Cripe, N. Aggarwal, R. Lanza et al., Nature (London) 568, 364 (2019).

[43] M. Yap et al., Nat. Photonics 568 (2019).

[44] H. J. Kimble, Y. Levin, A. B. Matsko, K. S. Thorne, and S. P. Vyatchanin, Phys. Rev. D 65, 022002 (2001).

[45] E. Oelker, T. Isogai, J. Miller, M. Tse, L. Barsotti, N. Mavalvala, and M. Evans, Phys. Rev. Lett. 116, 041102 (2016).

[46] M. Evans, L. Barsotti, P. Kwee, J. Harms, and H. Miao, Phys. Rev. D 88, 022002 (2013).

[47] J. Miller, L. Barsotti, S. Vitale, P. Fritschel, M. Evans, and D. Sigg, Phys. Rev. D 91, 062005 (2015).

[48] E. Genin, M. Mantovani, G. Pillant, C. D. Rossi, L. Pinard, C. Michel, M. Gosselin, and J. Casanueva, Appl. Opt. 57, 9705 (2018).

[49] L. Barsotti et al., https://dcc.ligo.org/LIGO-T1800042/ public (2018).

[50] R. Lynch, S. Vitale, L. Barsotti, S. Dwyer, and M. Evans, Phys. Rev. D 91, 044032 (2015).

[51] A. Torres-Rivas, K. Chatziioannou, A. Bauswein, and J. A. Clark, Phys. Rev. D 99, 044014 (2019). 